\title{
A RULE BASED FUZZY SYNTHETIC EVALUATION METHOD FOR RISK ASSESSMENT IN PIPELINE TRANSPORT
}

\author{
Yutao Liu, Hao Hu, Di Zhang \\ School of Naval Architecture, Ocean and Civil Engineering, \\ Shanghai Jiao Tong University, China
}

Submitted 4 June 2013; resubmitted 27 January 2013, 19 February 2013; accepted 7 March 2013

\begin{abstract}
Integrity Management Program (IMP) for oil and gas pipeline system demands a robust risk assessment method, which needs fully utilize the expert knowledge on the empirical data and the ambiguous cause(s) - effect(s) failure mechanism and then keep a good balance between precision and practicality. A new method, Rule Based Fuzzy Synthetic Evaluation (RB-FSE) which combines Fuzzy Synthetic Evaluation (FSE) with Fuzzy Logic (FLo) is proposed in this paper. It is applied to the pipeline risk assessment for Third-Party Damage (TPD). The proposed method is compared with scoring-type method in a case study. Results indicate that the relative assessment values from RB-FSE model could better support risk-ranking and decision-making in the IMP.
\end{abstract}

Keywords: pipeline transport; integrity management program; fuzzy synthetic evaluation; fuzzy logic; third-party damage.

\author{
Abbreviations \\ AL - Activity Level; \\ API - American Petroleum Institute; \\ ASME - American Society of Mechanical Engineers; \\ CCM - Chain Comparison Method; \\ FIS - Fuzzy Inference System; \\ FL - Failure Likelihood; \\ FLo - Fuzzy Logic; \\ FSE - Fuzzy Synthetic Evaluation; \\ HAL - Hazardous Activity Level; \\ HCAs - High Consequence Areas; \\ IM - Integrity Management Program; \\ MCDM - Multi-Criteria Decision-Making; \\ PHSMA - Pipeline and Hazardous Materials Safety Ad- \\ ministration: \\ PM - Preventive Measure; \\ RB-FSE - Rule Based Fuzzy Synthetic Evaluation; \\ SA - Security Attribute; \\ TPD - Third-Party Damage.
}

\section{Introduction}

Since pipelines were generally recognized to be the safest and the most economical mode to transport hazardous substances, especially a liquid or gas in large quantities, still it may confront leakages, ruptures and even bursts that not only cause transportation interruption, product reduction, and an arduous work of clean-up operation etc., but more seriously pose catastrophic health, environment and safety accidents. As a proactive safeguard strategy to develop an optimized inspection and maintenance program, IMP for oil and gas pipeline has been highly emphasized by many countries in recent years. Based on relevant standards (API Standard 1160-2001; ASME B31.8S-2004) and practical experience (Liu et al. 2011; Liu, Hu 2011), a general flow of IMP for pipeline system is depicted as Fig. 1. Marked with the bold print

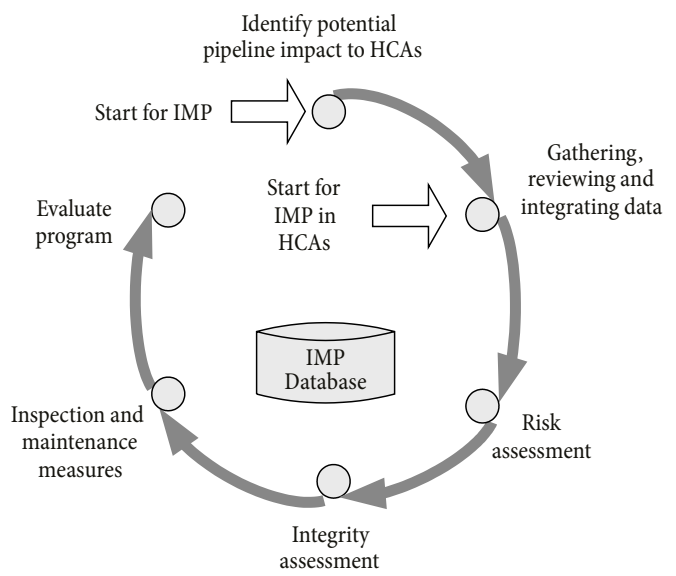

Fig. 1. Flow chart of IMP 
in Fig. 1, risk assessment plays a crucial role in IMP, because the results from it serve as the main basis to judiciously divide resources for inspection and maintenance among different pipelines, segments, or assets (Kishawy, Gabbar 2010).

In pipeline industry, risk analysis, commonly composed by likelihood and consequence, can be carried out qualitatively, semi-quantitatively, and quantitatively. Qualitative analysis methods, also sometimes called scoring-type methods, have some dazzling merits like facile handling, low cost etc.; therefore, they have been widely applied in risk assessment, and among them the conspicuous one is the Pipeline Hazard Index Method putted forward by Muhlbauer (2004). However, with the changes in legislation in many countries requiring operators to use risk assessment in HCAs, new methodologies (Palmer-Jones et al. 2006; Bai, Y., Bai, Q. 2005; Willcocks, Bai 2000; DeWolf 2003) ranged from point scoring qualitative schemes to detailed quantified systems. Despite giving much more reliable results, the quantitative risk analysis with absolute probability values, unfortunately, was sometimes a daunting dilemma for engineers in IMP practice owing to the scarcity of precise data and the vagueness of the cause-effect mechanism. Therefore, many researches turned to use semiquantitatively methods, especially those taking account of fuzzy techniques. Two primary ways to utilize fuzzy techniques were:

- the combinations of fuzzy techniques with commonly used risk assessment methods, such as fault tree analysis and analytic hierarchy process (Yuhua, Datao 2005; Rajani et al. 2006; Markowski, Mannan 2009);

- the hierarchical structures with a series of fuzzy inference processes (Singh, Markeset 2009; Doremami et al. 2010; Liu, Hu 2012).

These researches guaranteed that fuzzy techniques, especially FSE and FLo, were rational and available mathematic tools to tackle the imprecise yet important data and the vague accident mechanism widely existing in pipeline engineering. However, it was insufficient to reflect the mutual relationship among the non-independent evaluation indexes by a simple weighting process in the FSE, while it might be hard-sledding and timeconsuming to establish many fuzzy inference processes by FLo in a hierarchical structure for risk assessment.

With respect to pipeline damage, many incident causes have been reported. Historical data (PHMSA 2012) illustrated that a large number of pipeline incidents were caused by material/welding/equipment failure (25.7\%), excavation damage (19.7\%) and corrosion (17.9\%), as shown in Fig. 2. TPD, as the term used here, refers to any accidental damage caused by people not associated with the pipeline, mainly including excavation damage and other outside force damage (7.1\%), but excluding natural force damage. Some 20 to $30 \%$ of all pipeline failures in most time periods were attributed to TPD, and it was often the top priority in pipeline protection. In this paper we chose TPD as our application

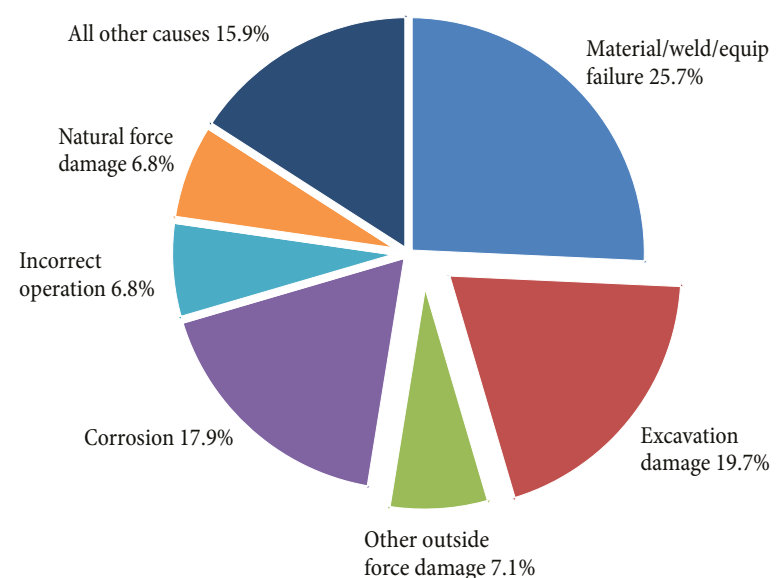

Fig. 2. Classification of the causes of incidents at pipelines in United States (1992-2011)

topic. Then a detailed analysis of TPD is presented in Section 2.1.

In order to keep a good balance between precision and practicality, this paper proposes a RB-FSE method for the pipeline risk assessment by a fine combination of FSE and FLo. The remainder of this paper is structured as follows: Section 1 briefly introduces FSE and FLo and then proposes the method of RB-FSE. Next, the RB-FSE model for TPD is established in Section 2. Section 3 presents the case study of a length of pipeline. RB-FSE model and scoring-type method are respectively employed in this case study, and the results from them are compared and discussed. Finally, conclusion is provided in last section.

\section{Proposed Methodology}

\subsection{Fuzzy Synthetic Evaluation}

FSE is often categorized as a special MCDM technique used by decision-makers to solve problems of conflicting multiple criteria. FSE is employed in this paper to translate various independent sub-indicators to one single comprehensive indicator directly related to the failure process.

\subsection{Fuzzy Logic}

A FLo based model uses a set of if-then rules and logical operators to establish a relationship between the input variables and the outputs. As shown in Fig. 3, this kind of model often consists of four components: fuzzy rule base, fuzzy inference process, fuzzification and defuzzification.

The rules of inference involved in the FLo model could be utilized for deriving truths state or proven truths (Ross 2010). So we could express the inherent failure mechanism in the form of fuzzy rules and then gather them as a fuzzy rule base so as to support the inference process. In this study, it is by the FLo that the comprehensive indicators representing the development stages of failure are synthesized to derive the final result. 


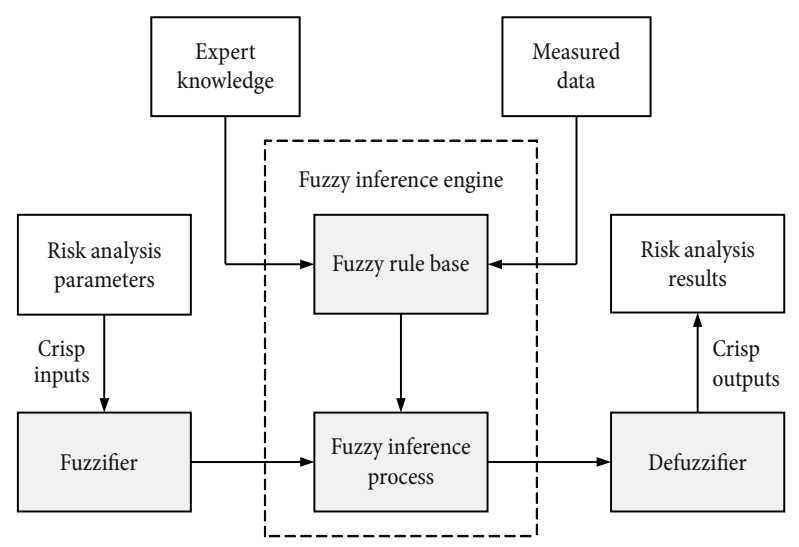

Fig. 3. An overview of the FLo system

\subsection{Rule-Based Fuzzy Synthetic Evaluation Method}

Both based on the fuzzy set theory, FSE and FLo contain the similar procedures - fuzzification and defuzzification, where crisp values and fuzzy sets could be transformed reciprocally. And a good combination of them could integrate their functions and form a systematical assessment method to handle fuzzy information. Following this speculation, the RB-FSE method is proposed as shown in Fig. 4. The detailed description of RB-FSE method is given with the risk assessment model of the TPD in the Section 2.

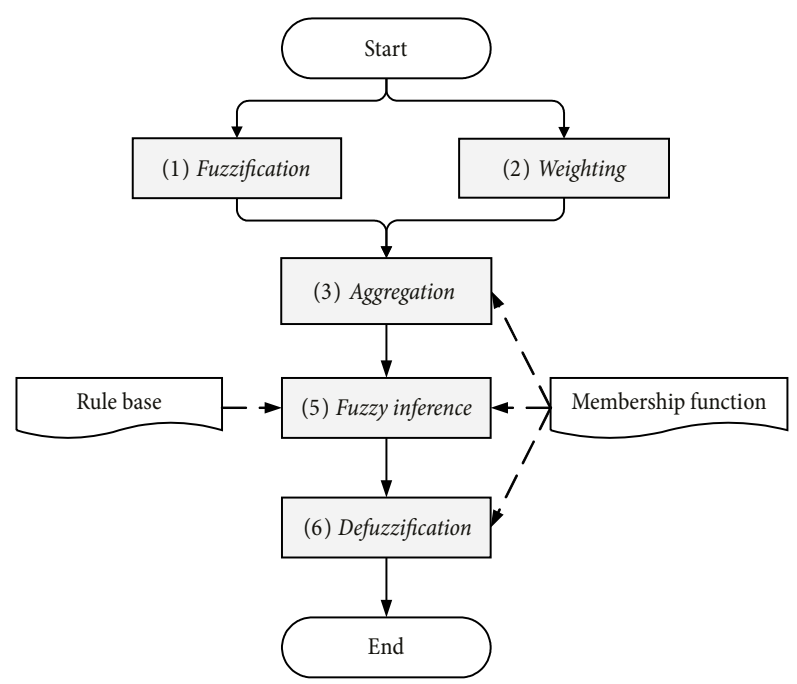

Fig. 4. Flowchart of RB-FSE method

\section{Risk Assessment for the Third-Party Damage to Pipeline System}

\subsection{Description of Third-Party Damage}

Combining the indicator system proposed by Muhlbauer (2004) and the feedback information from risk assessment practice in China, the cause-effect process with the influence factors of TPD could be depicted by Fig. 5. It is apparent that three indicators - Activity Level $(A L)$, Preventive Measure (PM), and Security Attribute (SA), directly result in the failure of pipeline. The explanations of them are given as follows:

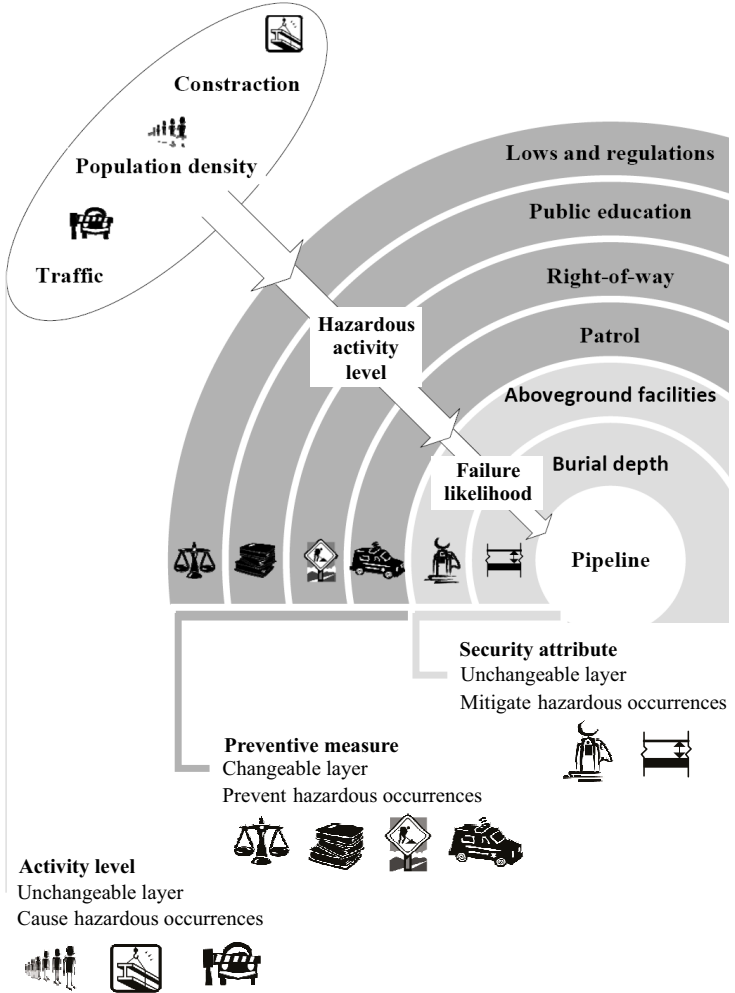

Fig. 5. Cause-effect process of TPD

- AL - for an analysis of the TPD potential, the area of opportunity is strongly affected by the level of activity near the pipeline;

- PM - some common PMs could effectively keep off or slow down the possible third-party activities risking the pipeline;

- SA - in order to resist the TPD, the engineers have designed some SAs, which cannot be easily changed during the operation of the pipeline system.

AL could be regarded as the precondition of the TPD, while $P M$ and $S A$ serve to reduce the failure risk of pipeline. Hazardous Activity Level (HAL) could be drawn from a combined consideration of $\mathrm{AL}$ and $\mathrm{PM}$, and then the Failure Likelihood (FL) could be estimated according to HAL and SA.

As shown in Table 1, two-level indicator system is then established:

- the 1st level contains three indicators that have been introduced above;

- the 2nd level includes eight sub-indicators that belong to their relevant indicators.

In the RB-FSE model, the 2nd-level indicators are transformed into the 1st-level indicators, and then they are integrated into a fuzzy inference to get the final result.

\subsection{Fuzzification of Raw Data}

It is assumed that every sub-indicator $S_{i, j}$ is associated with an underlying fuzzy set $F_{i, j}$, which is defined by $n$ linguistic constants and a specific universe of discourse, as shown in Table 1 . In this paper, $n=5$, that correspond 
to the condition states (very low, low, medium, high, very high) $[(v l, l, m, h, v h)]$ or (none, bad, medium, good, excellent $)[(n, b, m, g, e)]$. And it is specially provided all the linguistic variables are described as dimensionless quantities and normalized into a number between $[0,1]$ so as to avoid unnecessary dimensional conversion and calculation. Row data for a given sub-index $S_{i, j}$, whether crisp, fuzzy or predefined interval-valued, are mapped (or fuzzified) into $F_{i, j}$ in terms of the expertise with the universe of discourse. For example, supposing among ten experts four choose medium and the other six choose good, the fuzzy set $(0,0,0.4,0.6,0)$ of $(n, b, m, g, e)$ is used to replace 2 times/week to represent patrol frequency.

\subsection{Weighting and Aggregation}

Relative degrees of influence (importance or evidential credibility) have to be assigned in order to aggregate 2nd-level indicators towards 1st-level indicators. Let $W_{i, j}$ denote the relative weight of 2 nd-level indicators $i$ towards 1st-level indicators $j$. Weight values are obtained through expert opinion by CCM - a method based on pairwise comparison judgment matrix.

The weights of all sub-indicators within a comprehensive indicator are normalized to a sum of 1 prior to the application of the aggregation steps. The aggregation process is given by:

$$
I_{j}=\left[W_{1, j} \ldots W_{i, j} \ldots W_{N_{j}, j}\right] \cdot\left[F_{1, j} \ldots F_{i, j} \ldots F_{N_{j}, j}\right]^{T},
$$

where: '.' represents matrix multiplication. Since $F_{i, j}$ are 5 -tuple fuzzy sets and $W_{i, j}$ are scalars, is in fact a 5-tuple fuzzy set that represents the aggregated contribution of indicator $j$ towards the final result.

\subsection{Fuzzy Inference Systems}

In this work, two FISs are thereby designed respectively for the assessment of HAL and FL. The FISs are all constructed in accordance with the components of FLo introduced above. We take the FIS of $H A L$ for example.
Firstly, these linguistic variables $(A L, P M$, and $H A L)$ are decomposed with fuzzy variables $[\overline{A L}(v l, l$, $m, h, v h) ; \overline{P M}(n, b, m, g, e) ; \overline{H A L}(v l, l, m, h, v h)]$. And the calculation results of $A L$ and $P M$ form above steps could be inputted.

Next, membership functions are built and adapted. Out of several existing approaches, a fixed center-based membership function approach (Zhou et al. 1997) is adopted in this model, and symmetric Gaussian curves are used to describe fuzzy variables. The Gaussian membership function depends on two parameters and is given by:

$$
f(x, \sigma, c)=e^{\frac{-(x-c)^{2}}{2 \cdot \sigma^{2}}},
$$

where: $c$ is the mean value and is the standard deviation. Membership functions are centered, each at the mid-value of the numeric interval associated with each fuzzy variable. For example, the Gaussian membership function for the 'medium' of $A L$ is centered on the average value of the interval $(0.2,0.8)$ which corresponds to a mean value $c=0.5$, as shown in Fig. 5 . The standard deviation parameters for Gaussian functions are chosen such membership function curves are completed with the minimum and maximum points of the interval associated with each of the fuzzy variables. Based on this fixed center-based membership function approach, the membership functions for these linguistic variables are respectively modeled as fuzzy sets as shown in Fig. 6 .

Finally, the mapping between $A L, P M$ and $H A L$ is accomplished by the use of if-then rules, e. g., if $A L$ is high, and $P M$ is bad, then HAL is very high. For such a single attribute problem, a total of 13 if-then rules from expert knowledge are employed in this FIS to provide mapping between these linguistic variables, and the rule surface of this FIS is shown in Fig. 7.

The FIS for the assessment of $F L$ could be similarly established. The membership functions of the $\mathrm{SA}_{(}\left(I_{3}\right)$ and the $F L$ are modeled as shown in Fig. 8, and another 13 if-then rules are described by the rule surface shown in Fig. 9.

\begin{tabular}{|c|c|c|c|c|c|}
\hline $\begin{array}{l}\text { 1st-level } \\
\text { indicator }\end{array}$ & (j) & $\begin{array}{l}\text { 2nd-level } \\
\text { indicator }\end{array}$ & $(i, j)$ & Linguistic constants & Universe of discourse \\
\hline \multirow{3}{*}{$A L$} & \multirow{3}{*}{1} & Population density & 1,1 & $(v l, l, m, h, v h)$ & $\begin{array}{l}\text { (persons per square } \mathrm{km}\left(\mathrm{p} / \mathrm{km}^{2}\right)<1,1 \leq \mathrm{p} / \mathrm{km}^{2}<25 \text {, } \\
\left.25 \leq \mathrm{p} / \mathrm{km}^{2}<100,100 \leq \mathrm{p} / \mathrm{km}^{2}\right)\end{array}$ \\
\hline & & Construction & 2,1 & $(v l, l, m, h, v h)$ & (no, few, frequent) \\
\hline & & Traffic & 3,1 & $(v l, l, m, h, v h)$ & (no, rail or roadway traffic) \\
\hline \multirow{4}{*}{$P M$} & \multirow{4}{*}{2} & Right-of-way condition & 1,2 & $(n, b, m, g, e)$ & $\begin{array}{l}\text { (no, obstructed and not visible, partly unclear, clear } \\
\text { and unencumbered ) }\end{array}$ \\
\hline & & Public education & 2,2 & $(n, b, m, g, e)$ & (no, few, adequate, good and effective) \\
\hline & & Patrol & 3,2 & $(n, b, m, g, e)$ & (never, monthly, weekly, daily) \\
\hline & & Laws and regulations & 4,2 & $(n, b, m, g, e)$ & (no, not complete, complete and effective) \\
\hline \multirow[t]{2}{*}{$S A$} & \multirow[t]{2}{*}{3} & Minimum depth of cover & 1,3 & $(n, b, m, g, e)$ & $\begin{array}{l}\text { (depth }<0.5 \mathrm{~m}, 0.5 \mathrm{~m} \leq \text { depth }<1 \mathrm{~m}, \\
1 \mathrm{~m} \leq \text { depth }<1.5 \mathrm{~m}, 1.5 \mathrm{~m} \leq \text { depth })\end{array}$ \\
\hline & & Aboveground facilities & 2,3 & $(n, b, m, g, e)$ & (no, few with protection, many) \\
\hline
\end{tabular}

Table 1. Description of TPD indicators 


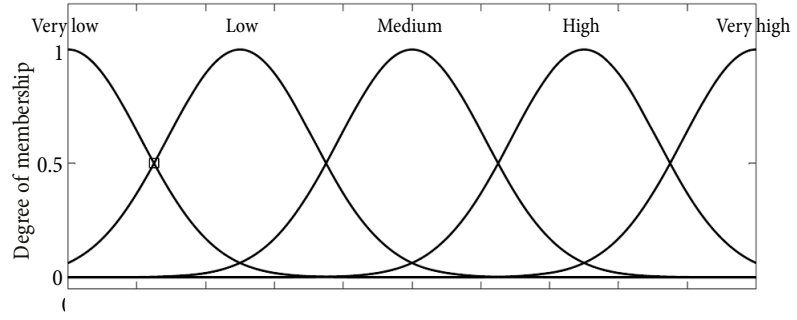

$A L$

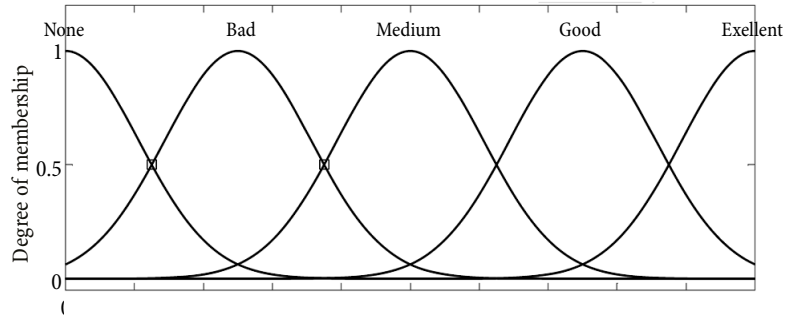

$P M$

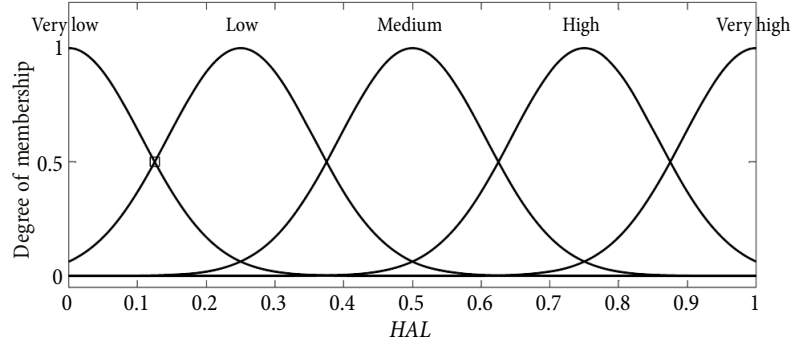

Fig. 6. The membership functions for $A L, P M$ and $H A L$

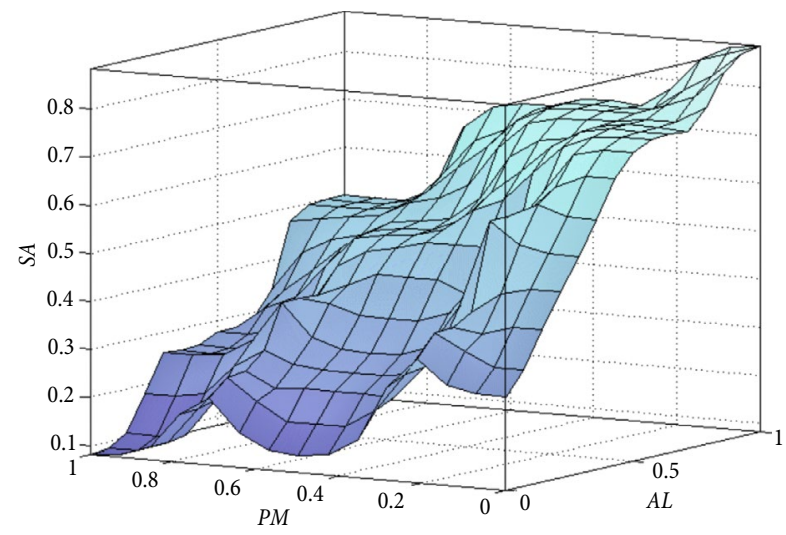

Fig. 7. Rule surface of FIS for the calculation of $H A L$

\subsection{Defuzzification}

The assessment results of $A L, P M, S A, H A L$ and $F L$ could all be transformed from fuzzy sets to crisp values through a defuzzification process. As one of the most computationally efficient methods, the height method is employed to defuzzify results from the aggregation process, and it is given by the algebraic expression:

$$
z^{*}=\frac{\sum \mu\left(z_{h}\right) \cdot z_{h}}{\sum \mu\left(z_{h}\right)}
$$

where: $\sum$ denotes the algebraic sum; $z_{h}$ and $\mu\left(z_{h}\right)$ are the max-value and the aggregation result of each membership function respectively. For example, if the aggrega-
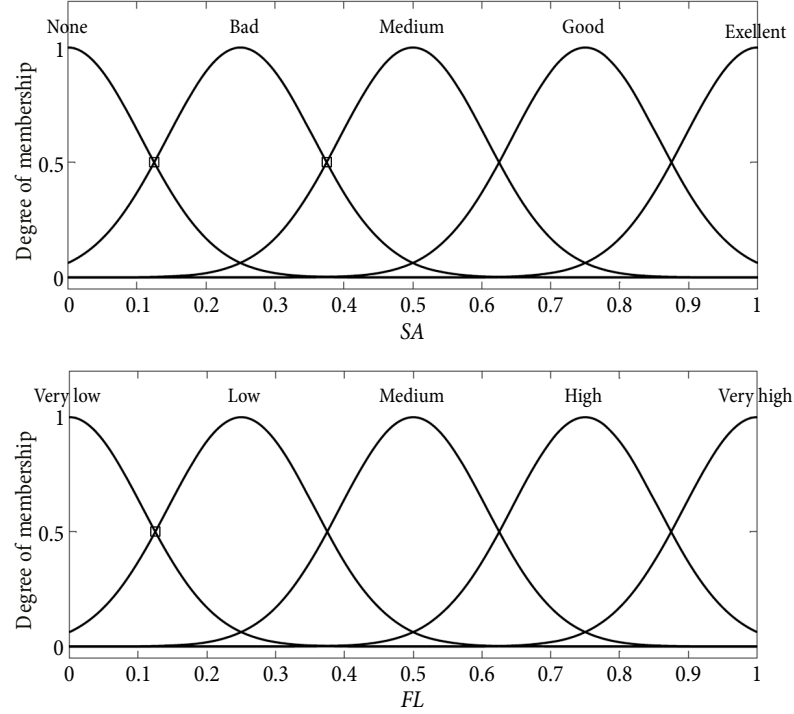

Fig. 8. The membership functions for the $S A$ and the $F L$

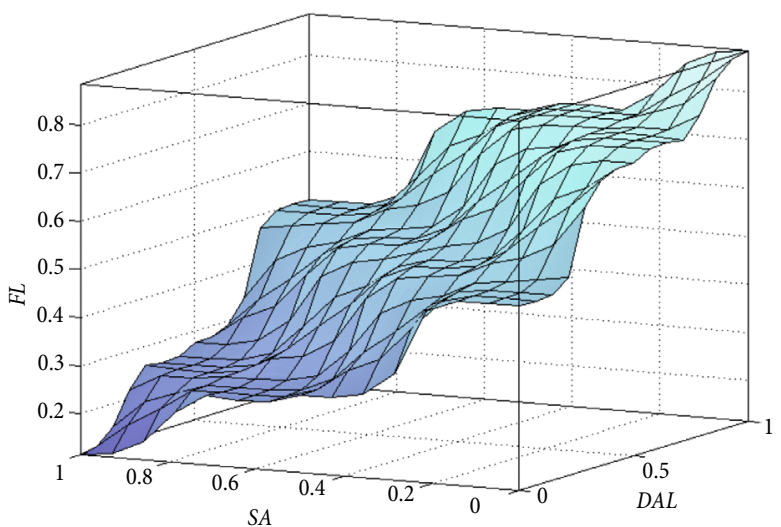

Fig. 9. Rule surface of FIS for the assessment of FL

tion result of $\overline{A L}\left(I_{1}\right)$ is $(0,0,0.85,0.15,0)$ and from the membership function shown in Fig. 6 the $\overline{Z_{h}}$ is $(0,0.25$, $0.5,0.75,1)$, then the crisp result of $A L$ is:

$$
z_{A L}^{*}=\frac{0.85 \cdot 0.5+0.15 \cdot 0.75}{0.85+0.15}=0.5375 \text {. }
$$

After going through all these steps above, the FISs for FL of pipeline for TPD is preliminarily established; nevertheless the membership function and the if-then rules in this model still need to be adjusted according to the practical feedback.

\section{Case Study}

\subsection{Case Description}

One case study cited from Wang et al. (2008) is presented here to illustrate and discuss the application of the RB-FSE model in FL assessment for the TPD. As shown in Fig. 10, the pipeline has lengths of $21.65 \mathrm{~km}$ and burial depths of $1.5 \mathrm{~m}$. It has been divided into 17 evaluation sections in terms of the geographical environment and the population density along the pipeline. 


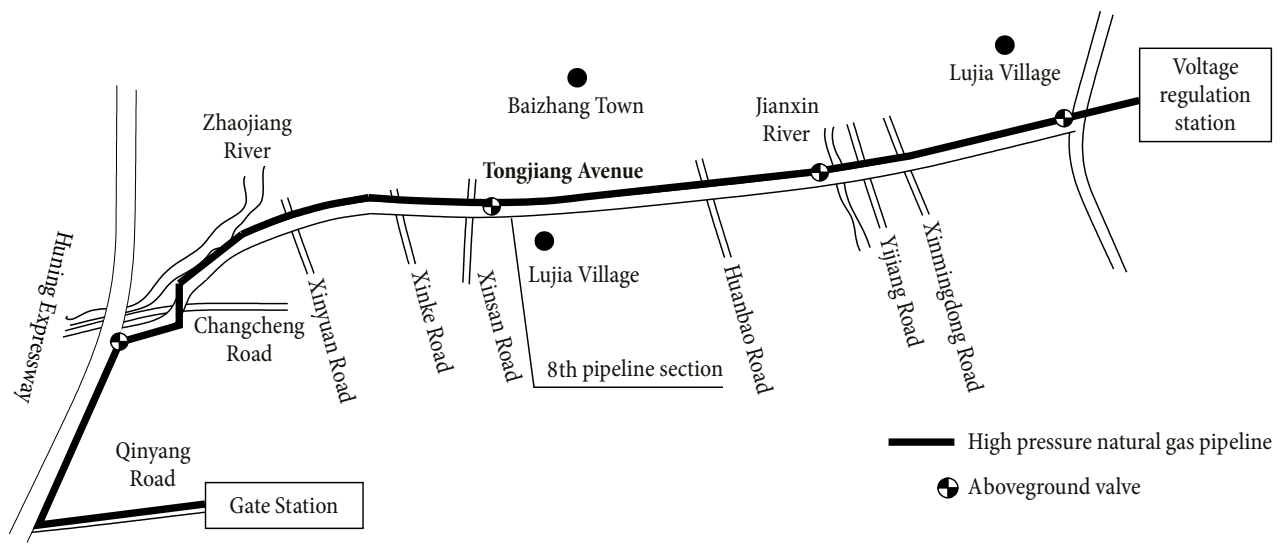

Fig. 10. The sketch of the high pressure natural gas pipeline

Among the whole pipeline, the 3rd, 8th, 12th and 16th sections are equipped aboveground valves but with good protection. A specialized pipeline operation company is responsible for the maintenance of the whole pipe, so all the sections enjoy almost the same prevention measures except for the right-of-way condition in a few sections where the caution signs are obstructed.

\subsection{Assessment for One Pipeline Section}

The calculation process using RB-FSE model could be illustrated by the example of the 8th pipeline section. The calculation results of sub-indicators and indicators are shown in the Table 2. Subsequently, after inputting the results of indicators into the FISs of $H A L$ and $F L$, the assessment result of $H A L$ is 0.498 and then the $F L$ is 0.303 . The assessment results of all 17 pipeline sections are procured using RB-FSE model and then represented in a scatter graph in Fig. 11.

\subsection{Comparison with the Scoring-type Method}

As the most common method in pipeline risk assessment, the scoring-type method is used here for comparison. The scoring-type method often uses expert grading in terms of an index system to generate relative scores of pipeline segments along a pipe route. Here we utilize the index system proposed by Muhlbauer (2004). The full score is 100 points, and a higher score means pipeline suffers higher likelihood of failure. Using the same indicator system and expert members, the final assessment results are shown in Fig. 12.

The $K$-means clustering method is utilized to rank these pipeline sections into different groups according to the assessment result of FL. To simplify the calculation, we assume the number of clusters is three and then gain the results of the cluster center, the cluster membership and the distance from assessment results to their relevant cluster center. The results of cluster analysis are also shown in Figs 11 and 12.

The average distance in each cluster is calculated and listed in the Table 3. For the purpose of facilitating comparison, the average distances from RB-FSE model have been multiplied by 100 so as to keep the same dimensions with the results from numerical scoring method.

In contrast with the numerical scoring method, it is apparent to find that RB-FSE model labels more pipeline sections with 'medium risk' and yet less sections with 'low risk' or 'high risk', which fits better with actual measurement. Moreover, in the same cluster the average distance from RB-FSE model is shorter than that from numerical scoring method. This suggests the results from RB-FSE model are more favorable in risk ranking and decision-making for their better diversity and stratification.

Table 2. The assessment results of sub-indicators and indicators for 8th pipeline section

\begin{tabular}{|c|c|c|c|c|c|}
\hline Sub-indicator & $\begin{array}{c}\text { Fuzzy-set-results } \\
(v l, l, m, h, v h) /(n, b, m, g, e)\end{array}$ & $\begin{array}{l}\text { Weight } \\
\text { value }\end{array}$ & Indicator & $\begin{array}{c}\text { Fuzzy-set-results } \\
(v l, l, m, h, v h) /(n, b, m, g, e)\end{array}$ & $\begin{array}{l}\text { Crisp } \\
\text { result }\end{array}$ \\
\hline Population density & $(0,0,1,0,0)$ & 0.5 & \multirow{3}{*}{$A L$} & \multirow{3}{*}{$(0,0,0.85,0.15,0)$} & \multirow{3}{*}{0.538} \\
\hline Construction & $(0,0,0.5,0.5,0)$ & 0.3 & & & \\
\hline Traffic & $(0,0,1,0,0)$ & 0.2 & & & \\
\hline Right-of-way condition & $(0,0,0,0,1)$ & 0.1 & \multirow{4}{*}{$P M$} & \multirow{4}{*}{$(0,0.3,0.3,0.3,0.1)$} & \multirow{4}{*}{0.550} \\
\hline Public education & $(0,0,1,0,0)$ & 0.3 & & & \\
\hline Patrol frequency & $(0,0,0,1,0)$ & 0.3 & & & \\
\hline Laws and regulations & $(0,1,0,0,0)$ & 0.3 & & & \\
\hline Minimum burial depth & $(0,0,0,0,1)$ & 0.65 & \multirow{2}{*}{$S A$} & \multirow{2}{*}{$(0,0,0.35,0,0.65)$} & \multirow{2}{*}{0.825} \\
\hline Aboveground facilities & $(0,0,1,0,0)$ & 0.35 & & & \\
\hline
\end{tabular}


Table 3. The comparison between RB-FSE model and numerical scoring method

\begin{tabular}{ll|l|l|c}
\hline \multirow{2}{*}{ Cluster } & \multicolumn{2}{c|}{ RB-FSE model } & \multicolumn{2}{c}{ Numerical scoring method } \\
\cline { 2 - 6 } & \multicolumn{1}{c|}{ Section number } & Average distance $(\times 100)$ & \multicolumn{1}{c}{ Section number } & Average distance \\
\hline 1 & $1,2,4$ & 0 & $1,2,4,5$ & 0.75 \\
\hline 2 & $3,5,6,7,9,10,11,13,14,15,17$ & 1.020 & $3,6,7,9,10,13$ & 2.667 \\
\hline 3 & $8,12,16$ & 0.333 & $8,11,12,14,15,16,17$ & 1.633 \\
\hline
\end{tabular}

Notes: 1 - low risk sections; 2 - medium risk sections; 3 - high risk sections.

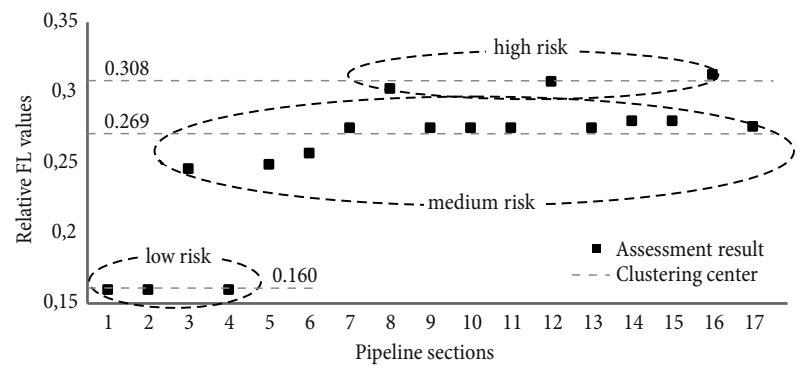

Fig. 11. The assessment results from RB-FSE model

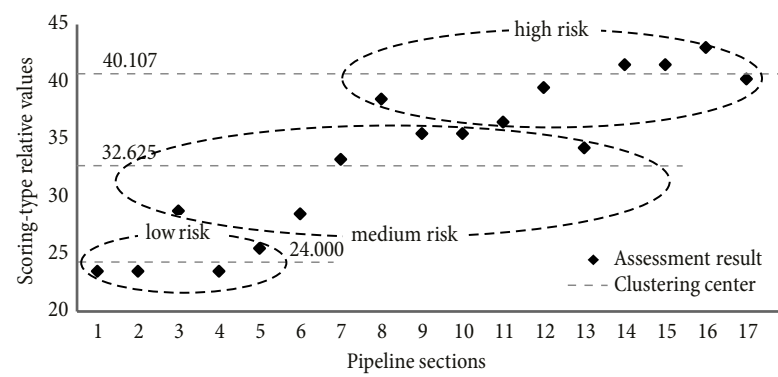

Fig. 12. The assessment results from numerical scoring method

\subsection{Decision-Making}

For the sake of reducing the FL of these pipeline sections with 'high risk', a feasible and effective method is to increase the patrol frequency in these areas. After the supplementary of special inspectors who are responsible for patrolling the high risk sections once a day, the reassessment results of these sections, as shown in Fig. 13, are then decreased to the acceptable values.

\section{Conclusions}

This paper proposed a semi-quantitative risk analysis method named RB-FSE, which combined fuzzy synthetic evolution and FLo. Therefore, fuzzy information including empirical data and ambiguous mechanism could be rationally and effectively utilized by fuzzy techniques in this method. TPD, as a common incident cause to pipeline, is described and analyzed. This is then followed by the creation of RB-FSE model to analyze the FL of pipeline suffering TPD. Subsequently, a length of pipeline is respectively evaluated by the RB-FSE model and scoring-type method. The results from these two methods are compared by $K$-means clustering method. Discussion results show RB-FSE model could be more conducive to support risk ranking and decision-making. Finally, the results from RB-FSE model are used to

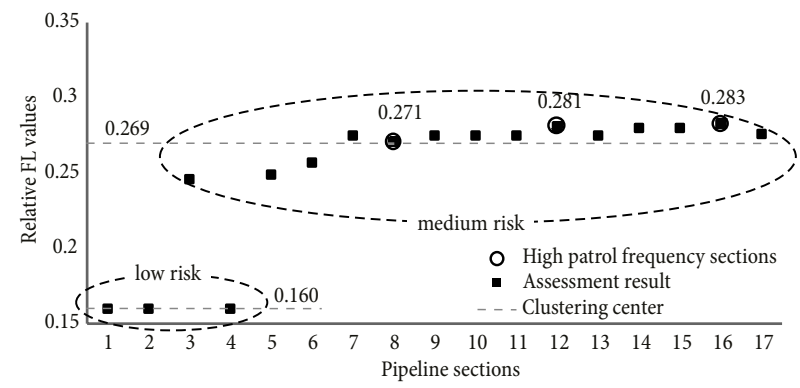

Fig. 13. The assessment results after increasing the patrol frequency

make a decision to eliminate 'high risk' pipeline sections, which present the application value of RB-FSE model.

The RB-FSE model could also enlarge the appliance-bound of fuzzy techniques. Besides what is illustrated in this paper - FL assessment of the TPD for pipeline, this model could be applied in other assessment problems for complex system. We will continue to develop this model into a vigorous tool to support IMP in our future research.

\section{Acknowledgements}

The first author gratefully acknowledges the support of the China Scholarship Council.

\section{References}

API Standard 1160-2001. Managing System Integrity for Hazardous Liquid Pipelines.

ASME B31.8S-2004. Managing System Integrity of Gas Pipelines.

Bai, Y.; Bai, Q. 2005. Subsea Pipelines and Risers. 2nd edition. Elsevier Science. $840 \mathrm{p}$.

DeWolf, G. B. 2003. Process safety management in the pipeline industry: parallels and differences between the pipeline integrity management (IMP) rule of the office of pipeline safety and the PSM/RMP approach for process facilities, Journal of Hazardous Materials 104(1-3): 169-192. http://dx.doi.org/10.1016/j.jhazmat.2003.08.008

Doremami, N.; Afshar, A; Mohammadi, A. D. 2010. Hierarchical risk assessment in gas pipelines based on fuzzy aggregation, in 2nd International Conference on Reliability, Safety \& Hazard (ICRESH-2010), 14-16 December 2010, Mumbai, India, 631-636. http://dx.doi.org/10.1109/ ICRESH.2010.5779626

Kishawy, H. A.; Gabbar, H. A. 2010. Review of pipeline integrity management practices, International Journal of Pressure Vessels and Piping 87(7): 373-380. http://dx.doi.org/10.1016/j.ijpvp.2010.04.003 
Liu, Y; Hu, H. 2012. Application of fuzzy logic to improve pipeline transport management, in ICPTT 2012: Better Pipeline Infrastructure for a Better Life, 19-22 October 2012, Wuhan, China, 1227-1239. http://dx.doi. org/10.1061/9780784412619.125

Liu, Y.; Hu, H. 2011. Functional modeling for an integrated pipeline integrity management system using IDEF0 approach, in ICTIS 2011: Multimodal Approach to Sustained Transportation System Development: Information, Technology, Implementation, 30 June - 2 July 2011, Wuhan, China, 1848-1855. http://dx.doi.org/10.1061/41177(415)233

Liu, Y.-T.; Hu, H.; Song, Y.-B. 2011. Pipeline integrity management system based on dynamic risk assessment, Journal of Shanghai Jiaotong University (Science) 45(5): 687-690 (in Chinese).

Markowski, A. S.; Mannan, M. S. 2009. Fuzzy logic for piping risk assessment (pfLOPA), Journal of Loss Prevention in the Process Industries 22(6): 912-927. http://dx.doi.org/10.1016/j.jlp.2009.06.011

Muhlbauer, W. K. 2004. Pipeline Risk Management Manual. 3rd edition. Gulf Professional Publishing. 395 p.

Palmer-Jones, R.; Turner, S; Hopkins, P. 2006. A new approach to risk based pipeline integrity management, in Proceedings of the 2006 International Pipeline Conference, Volume 2: Integrity Management; Poster Session; Student Paper Competition, 25-29 September 2006, Calgary, Alberta, Canada, 811-823. http://dx.doi.org/10.1115/IPC2006-10535

PHMSA. 2012. Pipeline Incidents Reports. US Department of Transportation's Pipeline and Hazardous Materials Safety Administration (PHMSA). Available from Internet: http:// primis.phmsa.dot.gov/comm/reports/safety/psi.html

Rajani, B.; Kleiner, Y.; Sadiq, R. 2006. Translation of pipe inspection results into condition ratings using the fuzzy synthetic evaluation technique, Journal of Water Supply: Research and Technology - Aqua 55(1): 11-24. http://dx.doi. org/10.2166/aqua.2005.069

Ross, T. J. 2010. Fuzzy Logic with Engineering Applications. 3rd edition. Wiley. $606 \mathrm{p}$.

Singh, M.; Markeset, T. 2009. A methodology for risk-based inspection planning of oil and gas pipes based on fuzzy logic framework, Engineering Failure Analysis 16(7): 20982113. http://dx.doi.org/10.1016/j.engfailanal.2009.02.003

Wang, K.-Q.; Wang, N; Zhang, C.; Lu, Z. 2008. Risk Characteristics of urban natural gas pipeline and improvement of the Kent method, China Safety Science Journal 18(9): 152-157.

Willcocks, J.; Bai, Y. 2000. Risk-Based inspection and integrity management of pipeline systems, in The Tenth International Offshore and Polar Engineering Conference, 28 May - 2 June 2000, Seattle, Washington, USA, 285-294.

Yuhua, D.; Datao, Y. 2005. Estimation of failure probability of oil and gas transmission pipelines by fuzzy fault tree analysis, Journal of Loss Prevention in the Process Industries 18(2): 83-88. http://dx.doi.org/10.1016/j.jlp.2004.12.003

Zhou, Q. Q.; Purvis, M; Kasabov, N. 1997. A Membership Function Selection Method for Fuzzy Neural Networks. Information Science Discussion Papers Series No 97/15. University of Otago, New Zealand. 6 p. Available from Internet: http:// otago.ourarchive.ac.nz/handle/10523/1027 